\title{
Impacto Econômico do Tratamento de Pacientes com Dengue no Brasil: uma Revisão Sistemática
}

\section{Economic Impact of Treatment of Patients with Dengue Fever in Brazil: a Systematic Review}

\author{
Paulo Roberto Haidamus de Oliveira Bastos ${ }^{\text {a }}$ Raquel da Silva Vieira*a
}

aUniversidade Federal de Mato Grosso do Sul, Programa de Pós-Graduação em Saúde e Desenvolvimento na Região Centro-Oeste. MS, Brasil.

*E-mail: raquel.vieira.biologia@hotmail.com

\begin{abstract}
Resumo
A dengue se tornou um problema crescente de saúde pública devido as altas taxas de mortalidade e morbidade, além de impor uma carga econômica substancial em um país em desenvolvimento. O presente estudo objetivou revisar de forma sistemática a literatura científica atual para sintetizar o efeito do impacto econômico do tratamento de pacientes com dengue no Brasil. A pesquisa foi realizada entre os meses de agosto a outubro de 2019, nos bancos de dados Medical Literature Analysis and Retrieved System (MEDLINE), Literatura Latino-americana e do Caribe em Ciências da Saúde (Lilacs) e Biblioteca Digital Brasileira de Teses e Dissertações (BDTD), totalizando uma amostra final de oito estudos. Diante dos achados, ficou evidente a escassez de estudos que abordam a temática do impacto na economia brasileira em consequência do tratamento de pacientes com dengue, revelando, a necessidade de investimentos em pesquisas científicas continuadamente, principalmente nas localizações geográficas distantes dos grandes centros de pesquisas científicas localizados na região Sudeste do país. O impacto do tratamento da doença no país é substancial, e, portanto, espera-se que este estudo possa estimular a expansão científica no país e orientar os formuladores de políticas para novas intervenções na saúde pública, a fim de diminuir a propagação da doença e minimizar dispêndios aos cofres públicos.
\end{abstract}

Palavras-chave: Custos e Análise de Custo. Assistência à Saúde. Dengue. Gastos em Saúde. Gestão em Saúde.

\begin{abstract}
Dengue has become a growing public health problem due to its high mortality and morbidity rates, as well as imposing substantial economic burden stemming from a developing country. The present study aimed to systematically review the current scientific literature to synthesize the economic impact effect of dengue patients treatment in Brazil. The research was performed between August and October 2019, in the Medical Literature Analysis and Retrieved System (MEDLINE), Latin American and Caribbean Literature in Health Sciences (Lilacs) and Brazilian Digital Library of Theses and Dissertations (BDTD), totaling a final sample of eight studies. In view of the findings, it was evident the scarcity of studies addressing the theme of the impact on the Brazilian economy as a result of the dengue patients treatment, revealing the need for investments in scientific research continuously, especially in the geographical locations distant from the large scientific research centers located in the Southeast region of the country. The impact of the disease treatment in the country is substantial, and therefore it is expected that this study can stimulate scientific expansion in the country and guide policymakers to new interventions in public health, in order to reduce the spread of the disease and minimize expenditures to public coffers.
\end{abstract}

Keywords: Costs and Cost Analysis. Health Care Delivery. Dengue. Health Expenditures. Health Management.

\section{Introdução}

A dengue é uma doença infeciosa viral causada pelo vírus (DENV) do gênero Flavivirus da família Flaviviridae. É transmitida aos seres humanos através da picada da fềmea de mosquitos do gênero Aedes, principalmente o mosquito Aedes aegypti (RODRIGUES et al., 2016).

Com ampla distribuição nas regiões tropicais e subtropicais no mundo, a doença atinge vários países causando a morte de milhares de pessoas anualmente (WORLD HEALTH ORGANIZATION, 2009). Os países pobres são os mais atingidos devido à má qualidade na infraestrutura, saneamento e falta de recursos no combate ao vetor, onde favorece a rápida dispersão dos vírus nas áreas urbanas (GOULD et al., 2017).

A dengue é uma doença de grande importância global para a saúde pública, pois afeta 2,5 milhões de pessoas em países endêmicos (WORLD HEALTH ORGANIZATION, 2009).
Recentemente foi observado a presença da doença em regiões não endêmicas dos Estados Unidos e Europa, onde os vírus se espalham através de viajantes infectados, favorecendo, assim o ciclo de transmissão (MARQUES-TOLEDO et al., 2019).

Foram estimados mais de 50 milhões de infecções por dengue nas Américas em 2010, onde cerca de 40\% ocorreram no Brasil. Sua incidência no país é alta e chegou a 401,6/100 mil habitantes em 2002. Em decorrência do elevado número de casos da doença, os custos com o tratamento também se elevaram. O custo com manejo no Brasil é o maior das Américas, o que corresponde a $42 \%$ dos custos totais relacionados à dengue no continente (BÖHM et al., 2016).

Dentre todos os países ocidentais endêmicos da doença, no período de 2000 a 2007, a média de custos médicos diretos chegaram a US\$ 1,35 bilhão/ano, além dos custos indiretos relacionados a perda da produtividade (OLIVEIRA; ITRIA; 
LIMA, 2019).

À vista disso, a dengue impõe carga econômica e social nos países de clima tropical, e dessa forma, estimar a carga que essa doença impõe ajuda a orientar os formuladores de políticas públicas a planejar as estratégias para gerenciar a doença (UNDURRAGA et al. 2015).

Em cinco países das Américas (Venezuela, El Salvador, Guatemala, Panamá e Brasil), e três países da Ásia (Malásia, Camboja e Tailândia), foram observados que a dengue impõe custos ao setor de saúde e economia, com despesas anual que pode ultrapassar US\$ 1,8 bilhão, levando em consideração os tratamentos, vigilância e controle de vetores (DONALISIO; FEITAS; ZUBEN, 2017).

A dengue gera impacto clínico negativo devido ao quadro febril inicial que pode progredir com as síndromes articulares e hemorrágicas. Esse quadro clínico leva os pacientes à incapacidade de suas atividades laborais (DONALISIO; FEITAS; ZUBEN, 2017) e gera um fardo econômico para as famílias de regiões endêmicas (LEGORRETA-SOBERANIS et al. 2017).

Assim, diante do exposto, torna-se relevante revisar a literatura científica acerca do efeito do impacto econômico do tratamento de pacientes com dengue no Brasil, bem como apresentar o estado da arte no país de forma a oferecer subsídios para pesquisas no país.

\section{Desenvolvimento}

\subsection{Metodologia}

Trata-se de um estudo de revisão sistemática conduzido a partir das recomendações propostas no guia Preferred Reporting Items for Systematic Reviews and MetaAnalyses (PRISMA) (MOHER, 2009), tendo como foco a análise da produção científica acerca do impacto econômico e social da dengue no Brasil, bem como, os custos médicos diretos e indiretos com tratamento da doença nos sistemas de saúde público e privado com base na questão norteadora: Qual o impacto econômico que o tratamento de pacientes com dengue gera ao Brasil?

As buscas foram realizadas nas bases de dados eletrônicas indexadas "Medical Literature Analysis and Retrieved System" (MEDLINE) via PubMed; na "Literatura Latino-americana e do Caribe em Ciências da Saúde" (Lilacs) via BVS e na Biblioteca e busca na biblioteca virtual de teses e dissertações "Digital Brasileira de Teses e Dissertações" (BDTD).

Foi utilizado descritores catalogados no Descritor em Ciências da Saúde - DeCS e no Medical Subject Headings - MeSH, nos idiomas inglês com seus termos similares em português: "Health Care Costs" (Custos de Cuidados Médicos Custos de Tratamento), "Sickness Impact Profile" (Perfil de Impacto da Doença), "Costs and Cost Analysis" (Custos e Análise de Custo), "Health Expenses" (Despesas em saúde), "Direct Service Costs" (Custos Diretos de Serviços), "Cost, health care" (Custo, cuidados de saúde), "Disease Impact Profile" (Perfil de Impacto da Doença), "Disease Charges"
(Taxas de Doença), "Hospitalization" (Hospitalização), todos acrescidos da palavra "dengue" (dengue) e "Brazil" (Brasil). Os descritores e os termos utilizados foram obtidos em seleção dos DeCs e MeSH nas bases Bireme e PubMed respectivamente para serem usados nas buscas, combinando operadores booleanos adequados. As buscas foram realizadas entre os meses de agosto e outubro de 2019. A seleção dos estudos foi realizada por dois pesquisadores, que inicialmente avaliaram os títulos e os resumos e posteriormente os textos foram lidos na íntegra e selecionados de acordo com critérios estabelecidos.

Os critérios de inclusão utilizados foram estudos que abordassem a temática do impacto econômico em decorrência do tratamento de pacientes com dengue nos sistemas de saúde público (Sistema Único de Saúde - SUS) e privado do Brasil, estudos que apresentavam os custos diretos ou indiretos associados ao tratamento da dengue, de forma relacionada, disponíveis na íntegra na web nos idiomas português, inglês e espanhol, publicados entre os anos de 2003 a 2019. Optouse por selecionar estudos a partir de 2003 devido ao ano de implementação do Programa Nacional de Controle da Dengue no Brasil (PNCD) em 2002 (BRASIL, 2002), quando foram intensificadas as ações de controle da doença no país. Estudos com foco nos custos de controle de vetores e revisões de literatura foram excluídos.

A partir dos estudos selecionados, realizou-se a tabulação dos dados que foram dispostos em um quadro as características gerais dos estudos e em duas tabelas as características específicas. No Quadro 1, foram considerados os seguintes itens: autor e ano de publicação, título, tipo de publicação, periódico ou fonte, fonte de pesquisa, região de realização dos estudos e limitações dos estudos. Nas Tabelas 1 e 2 foram agrupados os custos médicos diretos e indiretos da doença e os custos nos sistemas de saúde público e privado.

Foi construído um formulário estruturado no Microsoft Excel 2016 para padronizar os dados, incluindo as variáveis: custos diretos e indiretos para pacientes ambulatoriais e hospitalizados com dengue. Os custos médicos diretos são relacionados as despesas com diagnóstico, internação hospitalar e tratamento. Os custos indiretos relacionam-se a perda da renda média familiar e a perda do tempo de vida.

Para padronizar os resultados dos valores monetários calculados em dólares dos Estados Unidos (EUA) nos estudos selecionados, os valores foram convertidos em moeda local pela taxa de câmbio (1 Dólar dos Estados Unidos/USD = 4,1313 Real/BRL) do Banco Central do Brasil em 21 de outubro de 2019 (BANCO CENTRAL DO BRASIL, 2019).

\subsection{Resultados}

Após realizadas as buscas, o total de estudos encontrados no PubMed, Lilacs e BDTD foram 241, 15 e 134 publicações respectivamente. Desses, 81 estudos foram eliminados por duplicação, resultando em 309, dos quais, 182 foram excluídos através da leitura de títulos e resumos. Nessa etapa do estudo, dos 
127 estudos selecionados, 119 foram excluídos mediante critérios de inclusão e exclusão, restando 08 produções que compuseram o corpus deste estudo, conforme apresentado na Figura 1.

Figura 1 - Fluxograma PRISMA do processo de seleção das publicações no Brasil no período de 2003 a 2019. Campo Grande MS, Brasil

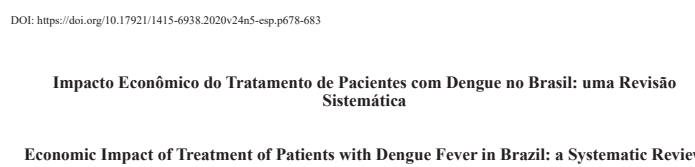

Economic Impact of Treatment of Patients with Dengue Fever in Brazil: a Systematic Review

Paulo Roberto Haidamus de Oliveira Bastosas" Raquel da Silva Vieira"

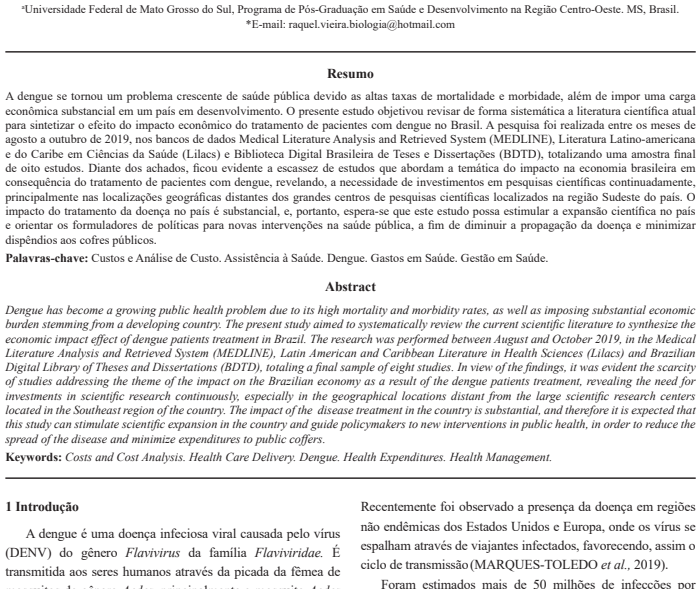

Fonte: Adaptado de Moher et al. (2009).
Quanto à qualidade dos estudos, os artigos foram classificados de acordo o Qualis (2019) da Coordenação de Aperfeiçoamento de Pessoal de Nível Superior (Capes). Dos seis artigos selecionados, cinco foram publicados em periódicos qualificados, entre B1 e A1 (Quadro 1). Em relação a abordagem metodológica, dos oito estudos selecionados, sete foram quantitativos e utilizaram as fontes de dados secundários mais comuns em pesquisas epidemiológicas, o Sistema de Informação Hospitalar (SIH), Sistema de Informação de Notificações de Agravos de Notificação (SINAN) (Quadro1).

No tocante as regiões brasileiras com produção científica, observa-se predomínio na região Sudeste com quatro estudos, sendo dois em Minas Gerais, um no Rio de Janeiro e um em São Paulo. No Centro-Oeste foi observado três estudos, sendo dois em Mato Grosso do Sul e um em Brasília; Nordeste apenas um estudo no Recife. Não foram encontrados nenhum estudo nas regiões Norte e Sul do país (Quadro 1).

Quanto as limitações apresentadas pelos autores na construção dos estudos, observou-se que a maioria delas estão relacionadas a impossibilidade de avaliar de forma absoluta os custos indiretos da dengue em decorrência do uso de dados secundários e possíveis vieses de informação (Quadro1).

Quadro 1 - Caracterização dos estudos selecionados para análise entre os anos de 2003 a 2019. Campo Grande MS, Brasil

\begin{tabular}{|c|c|c|c|c|c|c|}
\hline $\begin{array}{l}\text { Autor } \\
\text { (Ano) }\end{array}$ & Título & Publicação & $\begin{array}{l}\text { Periódico } \\
\text { /Fonte }\end{array}$ & $\begin{array}{c}\text { Fonte de } \\
\text { pesquisa de } \\
\text { dados }\end{array}$ & $\begin{array}{c}\text { Região } \\
\text { em } \\
\text { estudo }\end{array}$ & Limitação \\
\hline $\begin{array}{l}\text { Mendes } \\
(2003)\end{array}$ & $\begin{array}{l}\text { Mudanças climáticas e seus impactos } \\
\text { econômicos sobre a saúde humana: uma } \\
\text { análise da leishmaniose e da dengue no } \\
\text { Brasil }\end{array}$ & Tese & $\begin{array}{c}\text { Biblioteca Digital } \\
\text { Brasileira de Teses e } \\
\text { Dissertações (BDTD) }\end{array}$ & Secundário & $\mathrm{SE}$ & $\begin{array}{l}\text { Deficiência de } \\
\text { dados sobre } \\
\text { infraestrutura } \\
\text { e saneamento } \\
\text { básico. }\end{array}$ \\
\hline $\begin{array}{l}\text { Pereira et } \\
\text { al. }(2014)\end{array}$ & $\begin{array}{l}\text { Avaliação econômica dos casos de } \\
\text { Dengue atribuídos ao desastre de } 2011 \\
\text { em Nova Friburgo (RJ), Brasil }\end{array}$ & Artigo & $\begin{array}{l}\text { Ciência e Saúde } \\
\text { Coletiva (impresso) }\end{array}$ & Secundário & SE & $\begin{array}{l}\text { Fontes de dados } \\
\text { limitantes para o } \\
\text { problema real. }\end{array}$ \\
\hline $\begin{array}{l}\text { Machado } \\
\text { et al. } \\
(2014)\end{array}$ & $\begin{array}{l}\text { Custos Diretos da Hospitalização da } \\
\text { Dengue no Brasil: Sistemas de Saúde } \\
\text { Pública e Privada e Uso das Diretrizes } \\
\text { da OMS }\end{array}$ & Artigo & $\begin{array}{l}\text { Plos Neglected } \\
\text { Tropical Diseases }\end{array}$ & Secundário & $\mathrm{CO}$ & $\begin{array}{l}\text { Utilização de } \\
\text { banco de dados. }\end{array}$ \\
\hline $\begin{array}{l}\text { Martelli et } \\
\text { al. }(2015)\end{array}$ & $\begin{array}{l}\text { Impacto Econômico da Dengue: Estudo } \\
\text { Multicêntrico em Quatro Regiões do } \\
\text { Brasil }\end{array}$ & Artigo & $\begin{array}{l}\text { Plos Neglected } \\
\text { Tropical Diseases }\end{array}$ & Secundário & NE & $\begin{array}{l}\text { Subnotificação } \\
\text { ou escassez de } \\
\text { dados. }\end{array}$ \\
\hline $\begin{array}{l}\text { Leite } \\
(2015)\end{array}$ & $\begin{array}{l}\text { Impacto da Dengue no Brasil em período } \\
\text { epidêmico e não epidêmico: Incidência, } \\
\text { Mortalidade, Custo hospitalar e } \\
\text { Disability Adjusted Life Years (DALY). }\end{array}$ & Dissertação & $\begin{array}{c}\text { Biblioteca Digital } \\
\text { Brasileira de Teses e } \\
\text { Dissertações (BDTD) }\end{array}$ & Secundário & $\mathrm{CO}$ & $\begin{array}{l}\text { Vieses de } \\
\text { informações por } \\
\text { usar banco de } \\
\text { dados. }\end{array}$ \\
\hline $\begin{array}{l}\text { Araújo et } \\
\text { al. }(2017)\end{array}$ & $\begin{array}{l}\text { Aumento da carga de dengue no Brasil e } \\
\text { unidades federadas, } 2000 \text { e 2015: análise } \\
\text { do Global Burden of Disease Study } 2015\end{array}$ & Artigo & $\begin{array}{l}\text { Revista Brasileira de } \\
\text { Epidemiologia }\end{array}$ & Secundário & $\mathrm{SE}$ & \begin{tabular}{|l} 
Limitações de \\
informações nos \\
bancos de dados.
\end{tabular} \\
\hline $\begin{array}{l}\text { Abe \& } \\
\text { Miraglia } \\
(2018)\end{array}$ & $\begin{array}{l}\text { Incidência de dengue e custos associados, } \\
\text { nos períodos anterior (2000-2008) e } \\
\text { posterior (2009-2013) à construção das } \\
\text { usinas hidrelétricas em Rondônia }\end{array}$ & Artigo & $\begin{array}{l}\text { Epidemiologia e } \\
\text { Serviços de Saúde }\end{array}$ & Secundário & SE & $\begin{array}{l}\text { Ausência de } \\
\text { dados primários. }\end{array}$ \\
\hline $\begin{array}{l}\text { Machado } \\
\text { et al. } \\
(2019)\end{array}$ & $\begin{array}{l}\text { Segurança e custos das práticas de } \\
\text { transfusão de sangue em casos de dengue } \\
\text { no Brasil }\end{array}$ & Artigo & $\begin{array}{l}\text { Plos Neglected } \\
\text { Tropical Diseases }\end{array}$ & Secundário & $\mathrm{CO}$ & $\begin{array}{l}\text { Utilização de } \\
\text { banco de dados, } \\
\text { impossibilitando } \\
\text { avaliação de } \\
\text { custos indiretos. }\end{array}$ \\
\hline
\end{tabular}

Fonte: Dados da pesquisa. 
Dos principais achados dos custos médicos diretos do tratamento da dengue no Brasil, sete estudos estão diretamente relacionados aos tratamentos hospitalares, e três que abordaram os custos indiretos, estão fortemente relacionados a perda da renda familiar (Quadro 2).

Quadro 2 - Síntese panorâmica dos principais achados de custos médicos diretos e indiretos do tratamento de pacientes com dengue no Brasil. Campo Grande MS, 2019

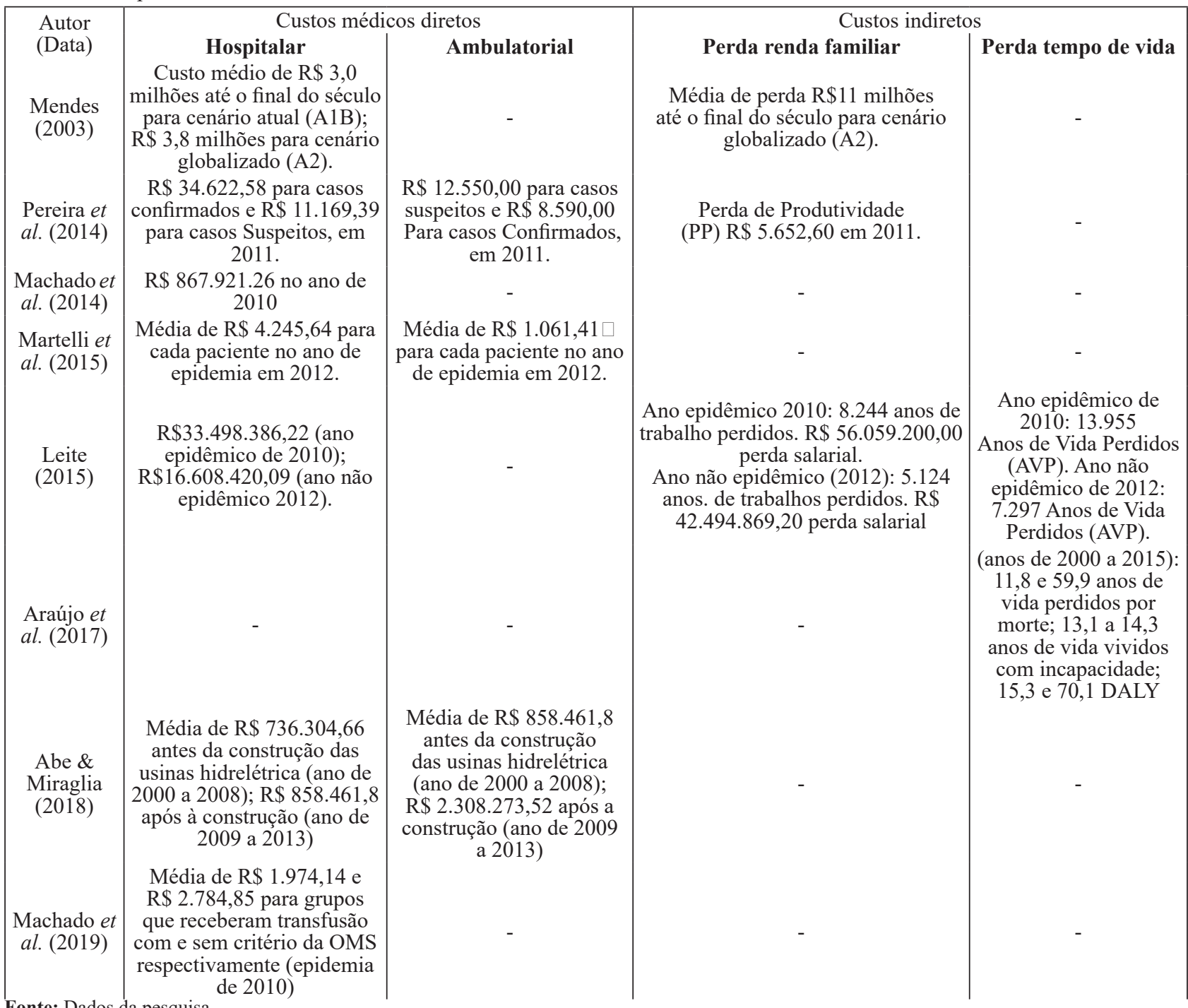

Fonte: Dados da pesquisa.

$$
\text { de 2010) }
$$

Com as epidemias de dengue no país, os custos com a ser mais onerosos, em relação ao serviço público, como tratamento da doença nos serviços de saúde privado chegam apresentados no Quadro 3.

Quadro 3 - Custos com tratamento de pacientes com dengue nos sistemas de saúde do Brasil. Campo Grande MS, 2019.

\begin{tabular}{|l|}
\hline \multicolumn{1}{|c|}{ Autor (Data) } \\
Mendes (2003) \\
Pereira et al. (2014) \\
Machado et al. (2014) \\
Martelli et al. (2015) \\
Leite (2015) \\
Araújo et al. (2017) \\
Abe \& Miraglia (2018) \\
Machado et al. (2019)
\end{tabular}

Fonte: Dados da pesquisa.

\section{Público}

Até o final do século: elevação de $\mathrm{R} \$ 85$ mil para $\mathrm{R} \$ 120$ mil em um cenário globalizado; R \$ 80 mil para R\$ 130 mil os custos com internações para um cenário não globalizado

$\mathrm{R} \$ 58.341,97$

$\mathrm{R} \$ 56.588,4$

Custos para cada caso de dengue: $\mathrm{R} \$ 9.304,89$

$$
\mathrm{R} \$ 50.106 .806,31
$$

R \$ 15,3 milhões anterior a construção; R $\$ 29,3$ milhões posterior $\mathrm{R} \$$ 75.191,19 em transfusão sem critérios da OMS; R\$ 23.689,68 em transfusão com critérios

\section{Privado}

Custos para cada caso de dengue: $\mathrm{R} \$ 26.105,73$ 


\subsection{Discussão}

Com base nos resultados obtidos, é evidente a escassez de estudos que abordam a temática no país, principalmente na região Norte do Brasil, onde a dengue se mantém como uma endemia e um grave problema de saúde pública.

O país possui enorme heterogeneidade espacial na distribuição das pesquisas científicas, concentrando-as na região Sudeste, com destaques aos Estados que sediam as universidades públicas historicamente consolidadas, como São Paulo e Rio de Janeiro, uma vez que a elas é atribuída a responsabilidade na maioria da produção científica no país (SIDONE, HADDAD; MENA-CHALCO, 2016). A vista disso, é notório que a desigualdade regional na produção científica pode estar fortemente associada a diferença na distribuição de recursos científicos e tecnológicos.

Constatou-se também que a maioria dos estudos realizados, utilizaram plataformas online de saúde de acesso gratuito, o que facilitou na obtenção de dados e possibilitou o conhecimento do perfil epidemiológico da população estudada. Entretanto, a qualidade e a confiabilidade das informações podem comprometer a fidedignidade dos dados devido a possibilidade de vieses (MACHADO; MARTINS; LEITE, 2016).

Com base nos estudos analisados, foi observado que os custos médicos diretos no sistema privado de saúde são mais onerosos. O valor de uma consulta ambulatorial no sistema privado pode custar até quatro vezes a mais que no SUS, e menor que o custo de uma internação (MARTELLI et al. 2015). Os valores são ainda maiores quando pacientes com trombocitopenia decorrente do agravamento da doença são hospitalizados para transfusão de hemoderivados ou necessitam de leitos de Unidade de Tratamento Intensivo (UTI) (MACHADO et al. 2019). O custo elevado no sistema de saúde privado também é observado em outras localidades do mundo onde a doença é endêmica, como a Índia, no qual os custos com hospitalizações podem ser 28 vezes maiores em hospitais privados, em relação aos públicos (BAJWALA et al. 2019).

No Brasil, essa discrepância provavelmente se deve ao fato de que o SUS transfere uma quantia média que varia de R\$ 303,57 a R\$ 385,19 para cada hospitalização, mediante Autorização de Internação Hospitalar (AIH) à hospitais credenciados (BRASIL, 2019). Os valores custeados pelo sistema público podem variar para mais quando há necessidade de exames complexos, como tomografia computadorizada, ressonância magnética, transfusões e leitos de UTIs (BRASIL, 2012).

Durante as epidemias, com aumento de casos sintomáticos, grande parcela da população busca atendimento ambulatorial, causando muitas vezes superlotação e aumento dos gastos no sistema público. Os achados aqui corroboram com o estudo de Oliveira, Ítria e Lima (2019), em que as epidemias de dengue impactam significativamente o sistema público de saúde por concentrar o maior número desses atendimentos.

Em virtude disso, Tasca et al. (2020), afirmam que o fortalecimento da Atenção Primária à Saúde (APS) no Brasil, com suporte tecnológico acessível, estrutura e financiamento adequado de recursos, bem como, campanhas de promoção da saúde são métodos que podem melhorar a qualidade de vida da população, além de reduzir custos e desafogar o nível secundário.

Além dos custos médicos diretos, foram observados aqui, que as epidemias de dengue geram custos para a sociedade. Os sintomas clínicos da doença fazem com que o indivíduo se ausente de suas atividades laborais (DONALÍSIO; FREITAS; ZUBEN, 2017). Quando a doença progride para forma grave, algumas síndromes neurológicas e pulmonares prolongam o tempo de ausência do trabalho, conhecido como absenteísmo, que na somatória dos dias afastados, impacta negativamente na renda familiar desse indivíduo (BRASIL, 2016).

A dengue é um problema de saúde pública global que causa morte e incapacita milhares de pessoas no mundo. $\mathrm{O}$ presente estudo mostrou que a doença gera carga substancial na saúde da população afetada, como encontrado nos estudos de Leite (2015) e Araújo et al. (2017). Esses autores avaliaram a carga substancial na saúde da população brasileira por meio de ferramentas de monitoramento em saúde, o Disability Adjusted Life Years (DALY) que medem o efeito da mortalidade e problemas de saúde na qualidade de vida da população (INSTITUTO DE MÉTRICA E AVALIAÇÃO EM SAÚDE, 2013). O DALYs é utilizado em pesquisas em todo o mundo para fornecer informações dos desafios que os profissionais de saúde e a população estarão prestes a enfrentar e assim auxiliam os tomadores de decisões na implementação de políticas públicas de saúde com base em evidências (INSTITUTO DE MÉTRICA E AVALIAÇÃO EM SAÚDE, 2013).

\section{Conclusão}

Os estudos evidenciaram o real cenário científico brasileiro acerca do impacto na economia brasileira em consequência do tratamento de pacientes com dengue, revelando, a necessidade de investimentos em pesquisas científicas continuadamente, principalmente nas localizações geográficas distantes dos grandes centros de pesquisas científicas localizados na região Sudeste do país.

A carga econômica do tratamento de pacientes com dengue no Brasil se mostrou substancial, com impacto significativo no sistema público de saúde, o qual remete grande parte dos atendimentos aos pacientes independente da classe social.

Os resultados aqui obtidos, possibilitaram refletir sobre a real situação epidemiológica dos países em desenvolvimento e o importante papel que o Estado tem em prover saúde e subsidiar recursos assistenciais com qualidade à população afetada, principalmente nas camadas menos favorecidas.

Embora, foram encontrados poucos estudos que 
abordassem a temática no país, todos foram consistentes no propósito aqui estabelecido, e afirma-se então, a importância desses dados para a tomada de decisões em saúde pública com medidas preventivas promissoras de controle da doença que envolva vários setores da sociedade, e não somente a área da saúde.

\section{Referências}

ABE, K.C.; MIRAGLIA, S.G.E.K. Incidência de dengue e custos associados, nos períodos anterior (2000-2008) e posterior (2009-2013) à construção das usinas hidrelétricas em Rondônia. Epidemiol. Serv. Saúde, v.27, n.2, 2018. doi: 10.5123/S167949742018000200012

ARAÚJO, V.E.M. et al. Aumento da carga de dengue no Brasil e unidades federadas, 2000 e 2015: análise do Global Burden of Disease Study 2015. Rev. Bras. Epidemiol. v.20, p.205-216, 2017. doi: 10.1590/1980-5497201700050017

BAJWALA, V.R. et al. Severity and costs associated with hospitalization for dengue in public and private hospitals of Surat city, Gujarat, India, 2017-2018. Trans. Rev. Soc. Trop. Med. Hyg., v.113, n.11, p.661-669, 2019. doi.org/10.1093/trstmh/trz057

BANCO CENTRAL DO BRASIL. Conversor de Moedas. Disponível em: https://www.bcb.gov.br/conversao. Acesso em: 21 out. 2019.

BÖHM, A.W. et al. Tendência da incidência de dengue no Brasil, 2002-2012. Epidemiol. Serv. Saúde, v.25, n.4, p.725-733, 2016. doi: 10.5123/S1679-49742016000400006

BRASIL, Ministério da Saúde. Fundação Nacional de Saúde (FUNASA). Programa Nacional de Controle da Dengue. Brasília: MS, 2002.

BRASIL, Ministério da Saúde. Agência Nacional de Saúde Suplementar (ANS). Caderno de Informações da Saúde Suplementar: beneficiários, operadoras e planos. Rio de Janeiro, 2012. Disponível em: http://www.ans.gov.br/images/stories/ Materiais_para_pesquisa/Perfil_setor/Caderno_informacao saude_suplementar/20130115_caderno_DEZEMB_revisado.pdf. Acesso em: 12 out. 2020.

BRASIL, Ministério da Saúde. Secretaria de Vigilância em Saúde. Departamento de Vigilância das Doenças Transmissíveis. Dengue: diagnóstico e manejo clínico: adulto e criança. Brasília: MS, 2016.

BRASIL, Ministério da Saúde. Valores médios das internações por dengue pelo SUS. Sistema de Informações Hospitalares do SUS (SIH/SUS). Disponível em: http://tabnet.datasus.gov.br/cgi / tabcgi.exe?sih/cnv/niuf.def. Acesso em: 10 out. 2020.

DONALISIO, M.R; FREITAS, A.R.R; ZUBEN, A.P.B.V. Arboviroses emergentes no Brasil: desafios para a clínica e implicações para a saúde pública. Rev. Saúde Pública, v.51, p.30, 2017. doi.org/10.1590/S1518-8787.2017051006889 1

GOULD, E. et al. Emerging arboviruses: why today? One Health, v.4, p.1-13, 2017. doi: 10.1016 / j.onehlt.2017.06.001

IHME - Instituto de Métrica e Avaliação em Saúde. Carga de doença global: gerando evidências, política de orientação. Seattle: IHME, 2013.

LEGORRETA-SOBERANIS, L.J et al. Household costs of dengue illness: secondary outcomes from a randomised controlled trial of dengue prevention in Guerrero state, Mexico. BMC Saúde Pública, v.17, p.411, 2017. doi: 10.1186/s12889-017-4304-x

LEITE, P. Impacto da Dengue no Brasil em período epidêmico e não epidêmico: incidência, mortalidade, custo hospitalar e Disability Adjusted Life Years (DALY). 2015. Disponível em: https://repositorio.unb.br/handle/10482/192 64. Acesso em: 11 set. 2020 .

MACHADO, A.A.V. et al. Direct costs of dengue hospitalization in Brazil: public and private health care systems and use of WHO Guidelines. PLoS Negl. Trop. Dis., v.8, n.9, 2014. doi: 10.1371/ journal.pntd.0003104

MACHADO, J.P; MARTINS, M; LEITE, I.C. Qualidade das bases de dados hospitalares no Brasil: alguns elementos. Rev. Bras. Epidemiol. v.19, n.3, p.567-581, 2016. doi: 10.1590/19805497201600030008

MACHADO, A.A.V. et al. Safety and costs of blood transfusion practices in dengue cases in Brazil. PLoS One, v. 14, n. 7, p. e0219287, 2019. doi: org/10.1371/journal.pone.0219287

MARQUES-TOLEDO, C.A.M. et al. Probability of dengue transmission and propagation in a non-endemic temperate area: conceptual model and decision risk levels for early alert, prevention and control. Parasit Vectors, v.12, p.1-38, 2019. doi: 10.1186/s13071-018-3280-Z

MARTELLI, C.M.T. et al. Economic impact of dengue: multicenter study across four brazilian regions. PLoS Negl. Trop. Dis. v.9, n.9, 2015. doi: 10.1371/journal.pntd.0004042

MENDES, C.S. Mudanças climáticas e seus impactos econômicos sobre a saúde humana: uma análise da leishmaniose e da dengue no Brasil. 2003. Disponível em: https://www.locus.ufv.br/ handle/123456789/155 Acesso: 12 set. 2020.

MOHER, D. et al. Preferred reporting items for systematic reviews and meta-analyses: the PRISMA Statement. PLoS Med., v.6, 2009. doi: 10.1371/journal.pmed.1000097

OLIVEIRA, L.N.S; ITRIA, A.; LIMA, E.C. Cost of illness and program of dengue: a systematic review. PLoS One, v.14, n.2, 2019. doi.org/10.1371/journal.pone.0211401

PEREIRA, C.A.R. et al. Avaliação econômica dos casos de Dengue atribuídos ao desastre de 2011 em Nova Friburgo (RJ), Brasil. Ciênc. Saúde Coletiva, v.19, n.9, p.3693-3704, 2014. doi. org/10.1590/1413-81232014199.01682014

RODRIGUES, N.C.P. et al. Temporal and spatial evolution of dengue incidence in Brazil, 2001-2012. PLoS One, v.11, n.11, p. e0165945, 2016. doi: 10.1371/journal.pone.0165945

SIDONE, O.J.G; HADDAD, E.A; MENA-CHALCO, J.P. A ciência nas regiões brasileiras: evolução da produção e das redes de colaboração científica. Transinformação, v.28, n.1, p.15-31, 2016. doi.org/10.1590/2318-08892016002800002

TASCA, R. et al. Recomendações para o fortalecimento da atenção primária à saúde no Brasil. Rev. Panam Salud Publica, v. 44, p. e4, 2020. doi: 10.26633/RPSP.2020.4

UNDURRAGA, E.A. et al. Economic and disease burden of dengue in Mexico. PLoS Negl. Trop. Dis., v.9, n.3, p. e0003547, 2015. doi: 10.1371/journal.pntd.0003547

WHO - World Health Organization. Dengue guidelines for diagnosis, treatment, prevention and control. 2009. Disponível em: https://www.who.int/tdr/publications/documents/denguediagnosis.pdf. Acesso em: 5 set. 2020. 